\title{
Bone mineral density and serum testosterone in chronically trained, high mileage $40-55$ year old male runners
}

\author{
K J MacKelvie, J E Taunton, H A McKay, K M Khan
}

\begin{abstract}
Objectives-To identify physical activity that is beneficial for the maintenance of bone strength with increasing age by examining the relation between bone mineral density (BMD) and chronic endurance training in men. BMD at the proximal femur, its subregions, and the lumbar spine, and serum testosterone were compared between two groups of long distance runners with more than 20 years of training experience and non-athletic controls. Methods-Runners $(\mathrm{n}=12)$ were divided into $(a)$ high volume runners $(n=7)$, running $64-80 \mathrm{~km}$ a week, and $(b)$ very high volume runners $(n=5)$, running more than $95 \mathrm{~km}$ a week, and compared with non-athletic male controls, exercising in non-endurance oriented activities two to four times a week. BMD $\left(\mathrm{g} / \mathrm{cm}^{2}\right)$ at the total proximal femur, femoral neck, trochanteric region, and lumbar spine was measured by dual energy $x$ ray absorptiometry. Total testosterone (nmol/1) and free testosterone (pmol/1) in serum were measured by radioimmunoassay from single fasting blood samples.
\end{abstract}

Results-Height, weight, and age (range = 40-55 years) were not significantly different between groups. The high volume runners had significantly higher BMD at the total proximal femur $(1.09(0.17) v 0.94$ $(0.056))$, femoral neck (0.91 (0.16) $v 0.78$ $(0.071))$, and trochanteric region $(0.85$ $(0.14) \quad v \quad 0.73(0.053))$ than controls $(p<0.05)$. The differences in BMD for the proximal femur between the very high volume runners and the other two groups were not significant. There was no difference in lumbar spine BMD, total testosterone, or free testosterone between groups. However, there was a significant negative correlation between total testosterone $(r=-0.73, p<0.01)$ and free testosterone $(r=-0.79, p<0.005)$ and running volume in the distance runners.

Conclusions-Long term distance running with training volumes less than $80 \mathrm{~km}$ a week had a positive effect on BMD of the proximal femur. With running volumes greater than $64 \mathrm{~km}$ a week, training was inversely related to testosterone levels, but levels remained within the normal range. (Br F Sports Med 2000;34:273-278)

Keywords: exercise; bone mineral density; male athletes; runners; endurance training; testosterone
Physical activity level is an important, modifiable influence on bone mineral density (BMD). For this reason, and given that high bone mass reduces the risk of osteoporotic fracture, it is essential to define the levels of physical activity that will optimise bone health. Mechanical loading of the skeleton has been positively associated with BMD in studies that compared male athletes from a number of different sports with less active controls. ${ }^{1-3}$

Evidence from animal ${ }^{4-6}$ and cross-sectional athlete $\mathrm{e}^{7-8}$ studies indicate that both the magnitude and volume of loading influence a site specific skeletal response. The bone strains associated with distance running, a highly popular weight bearing activity, are of less magnitude but greater volume than those of high impact sports or resistance training. Although some studies comparing male distance runners with less active controls have reported higher $\mathrm{BMD}^{9}$ or bone mineral content $(\mathrm{BMC})^{10}$ in the runners, others have found the opposite. ${ }^{11} 12$ The conflicting results may be a manifestation of the large range in age, training volume, and training years of the subjects.

Several studies reviewed elsewhere ${ }^{13}$ have documented training volume related alterations in the regulation of the hypothalamicpituitary-gonadal axis in male endurance athletes. ${ }^{14}{ }^{15}$ Chronic high mileage training in some studies has resulted in lower levels of circulating testosterone than in sedentary or less active men. ${ }^{16-18}$ There is accumulating evidence that, in runners, there may be a threshold at around $100 \mathrm{~km}$ a week, above which there are negative implications for testosterone regulation. ${ }^{13}$

Testosterone is an important determinant of BMD during growth and for the adult skeleton. ${ }^{19}$ Secondary osteoporosis could result from chronic suppression of testosterone levels with distance running. The relations between high and very high mileage distance running, $\mathrm{BMD}$, and testosterone regulation in men have not been defined, as there have been no studies of high mileage runners with 20 year running histories. Thus we examined the relation between training volume, serum testosterone, and BMD at the proximal femur and lumbar spine in two groups of runners (high volume and very high volume) with a minimum of 20 years of consistent running training and a normally active control group.

\section{Methods}

\section{DISTANCE RUNNERS}

Twelve competitive white male distance runners were recruited from a mailing list of male 
masters runners over the age of 40 provided by the provincial athletic organisation in British Columbia, Canada. These runners were registered with the cross country/road running/ track and field association, and had therefore competed in a race within the previous year. Fifty seven potential subjects were invited to enter the study if they met the following criteria: (a) age between 40 and 55 years; $(b)$ training at a minimum weekly running volume of 64 $\mathrm{km}$ a week for at least 20 years; (c) planning to compete in a running competition of at least 10 $\mathrm{km}$ within the year; (d) non-smoker. Of the 18 respondents, 12 men met the selection criteria. The high volume runners $(\mathrm{n}=7)$ reported a training volume of between 64 and $80 \mathrm{~km}$ a week, and the very high volume runners $(n=5)$ a volume of between 95 and $112 \mathrm{~km}$ a week. Subjects reported starting age of training, current and previous training volumes $(\mathrm{km}$ a week), and training hours in a questionnaire.

NON-ATHLETIC CONTROLS

Healthy white men were recruited and matched for age and weight with the distance runners. Inclusion criteria were (a) age between 40 and 55 years; (b) non-smoker; (c) participating in non-endurance type activities two to four times a week; (d) weight between 61 and $87 \mathrm{~kg}$. Twelve of 16 respondents met the entry criteria and were included.

The study was approved by the University of British Columbia clinical screening committee for research and other studies involving human subjects. All subjects provided written consent and completed the study.

\section{BONE DENSITOMETRY}

BMD of the proximal femur (total, femoral neck, trochanteric region) and the lumbar spine (L1-L4) was measured by dual energy $x$ ray absorptiometry (QDR 4500; Hologic Inc, Waltham, Massachusetts, USA). All scans were performed by the same registered technician. Proximal femur scans were performed on the non-dominant side. The in vitro precision value for our instrument was $0.5 \%$ at the lumbar spine.

HORMONE ANALYSIS

Subjects reported to the university medical centre between 0700 and 0900 after 12 hours of fasting and 36 hours without strenuous exercise. A single $10 \mathrm{ml}$ blood sample was collected from each subject by the same doctor and clotted at room temperature. Samples were then centrifuged at $3200 \mathrm{rpm}$ and $4^{\circ} \mathrm{C}$. Serum was separated from the rest of the sample and stored at $-70^{\circ} \mathrm{C}$ to await batch analysis. Hormone analyses were performed on serum, using competitive binding radioimmunoassays. Total testosterone was analysed using the ACS: 180 testosterone assay (Chiron Diagnostics, East Walpole, Massachusetts, USA). Sensitivity for this assay falls between testosterone concentrations of 0.35 and $52.0 \mathrm{nmol} / 1$, with a reported precision of $6.5 \%$ (coefficient of variation) within the normal physiological range for total testosterone. ${ }^{20}$ Free testosterone in serum was assessed using the Coat-A-Count free testosterone radioimmunoassay (Diagnostics Products, Los Angeles, California, USA). The kit is sensitive for free testosterone values ranging from 1.9 to $173 \mathrm{pmol} / \mathrm{l}$, and can detect as little as $0.52 \mathrm{pmol} / 1$ free testosterone. Precision values (coefficient of variation) of $3.8 \%$ for within run tests and $4.2 \%$ for run to run tests have been reported. ${ }^{21}$

\section{ANTHROPOMETRIC MEASURES}

Height (in $\mathrm{m}$, to the nearest $0.005 \mathrm{~m}$ ), weight in light clothing (in $\mathrm{kg}$, to the nearest $0.1 \mathrm{~kg}$ ), and sum of seven skinfolds (in $\mathrm{mm}$, to the nearest $0.1 \mathrm{~mm}$ ) including triceps, biceps, subscapular, suprailiac, abdominal, thigh, and medial calf were measured according to Canadian Physical Activity, Fitness and Lifestyle Appraisal (CPAFLA) guidelines. ${ }^{22}$ Body mass index (BMI, weight/height ${ }^{2}$ ) was calculated.

ASSESSMENT OF NUTRIENT INTAKE

Subjects completed a three day dietary record, which was analysed by the Foodsmart computerised dietary analysis system (Sasquatch Software, North Vancouver, British Columbia, Canada). Average daily total energy (kcal) and calcium (mg) intakes were calculated and compared.

\section{STATISTICAL ANALYSIS}

Differences between very high volume, high volume, and control groups were determined by one way analysis of variance, followed by Tukey's HSD post hoc analysis. Statistical significance was set at $p<0.05$. We examined the relation between $\mathrm{BMD}$, total/free testosterone, and other descriptive variables using Pearson's product-moment correlation coefficients.

Table 1 Physical and training characteristics of very high volume runners, high volume runners, and controls

\begin{tabular}{llll}
\hline & Very high volume & High volume & Controls \\
\hline $\mathrm{n}$ & 5 & 7 & 12 \\
Age (years) & $49.1(5.2)[41-52]$ & $48.0(4.3)[42-55]$ & $47.4(5.3)[40-55]$ \\
Weight $(\mathrm{kg})$ & $72.0(4.6)[64.5-89.4]$ & $72.1(8.7)[68.6-80.0]$ & $76.7(6.2)[68.9-86.2]$ \\
Height $(\mathrm{m})$ & $1.84(0.07)[1.77-1.92]$ & $1.81(0.05)[1.74-1.88]$ & $1.80(0.06)[1.71-1.90]$ \\
Body mass index $\left(\mathrm{kg} / \mathrm{m}^{2}\right)$ & $21.3(2.5)[18.7-24.6]$ & $22.1(2.8)[19.4-27.2]$ & $23.6(1.7)[21.1-26.1]$ \\
Sum of 7 skinfolds $(\mathrm{mm})$ & $64.4(17.7)^{\star}[40.3-85.9]$ & $68.8(27.9)^{\star}[32.8-106.3]$ & $107.0(25.0)[48.5-140.2]$ \\
Running volume (km/week) & $101(7.0)[96-112]$ & $69(6.0)[64-80]$ & $\mathrm{NA}$ \\
Training (hours/week) & $10.6(2.6)[8-15]$ & $9.1(1.9)[7.5-13]$ & $4.8(2.5)[2.5-10]$ \\
Weight training (hours/week) & $0.9(0.7)[0-2]$ & $0.7(0.9)[0-2]$ & $1.6(1.3)[0-4]$ \\
Calcium intake (mg/day) & $1090(310)[680-1580]$ & $1050(330)[620-1470]$ & $870(650)[310-2460]$ \\
Energy intake (kcal/day) & $2650(700)[1980-3500]$ & $2407(590)[1570-3110]$ & $2120(390)[1690-2620]$ \\
\hline
\end{tabular}

Data are expressed as mean (SD) [range]

*Significantly different from controls $(p<0.05)$.

NA, not applicable. 


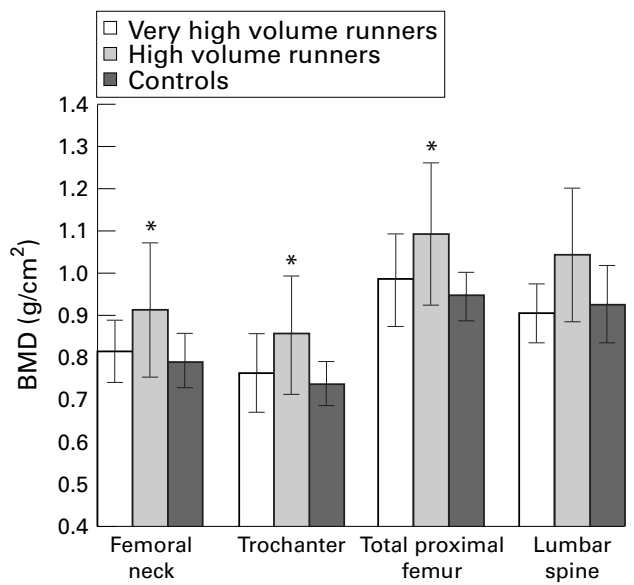

Figure 1 Mean bone mineral density (BMD) for very high volume runners, high volume runners, and controls. *Significantly higher than controls, $p<0.05$.

\section{Results}

SUBJECT CHARACTERISTICS

Table 1 summarises the physical characteristics, dietary intake, and general training characteristics of the subjects. Age, height, weight, and BMI did not differ significantly between groups. The sum of seven skinfolds was significantly higher in the control group $(\mathrm{p}<0.05)$. The minimum competitive distance in runners was $3000 \mathrm{~m}$ steeplechase $(n=1)$. All other runners had competed in $10 \mathrm{~km}$ events, nine had competed in marathons, and two in ultramarathons $(100 \mathrm{~km})$. All runners cross trained in other activities including cycling, swimming, and/or resistance training. Runners performed less than two hours of resistance training a week. Controls reported walking, hiking, resistance training, cycling, golfing, and tennis, but did not perform any endurance training. Nine control subjects resistance trained for two to four hours a week. Average daily calcium and energy intakes were not significantly different between groups (table 1).

BMD

High volume runners had significantly higher mean BMD than controls at the total proximal femur (1.09 (0.17) $v 0.94(0.056))$, femoral neck $(0.91 \quad(0.16) \quad v \quad 0.78 \quad(0.071))$, and trochanteric regions $(0.85 \quad(0.14) \quad v \quad 0.73$ (0.053)) (all p <0.05). The difference between high volume runners and controls at the lumbar spine was not significant $(1.04(0.16) v$ $0.92(0.095))$. The mean BMD for the very high volume runners was not significantly different from that of controls or high volume runners at any site: total proximal femur $(0.98$ (0.11)), femoral neck (0.81 (0.074)), trochanteric region $(0.76(0.093))$, and lumbar spine $(0.90(0.070))$ (fig 1$)$.
At the femoral neck, trochanter, and total proximal femur, all three group means were within 1 standard deviation of same age BMD $\mathrm{z}$ scores provided by the manufacturer of the dual energy $x$ ray absorptiometer (Hologic Inc). At the lumbar spine, all three group means were within 1.3 standard deviations of the reference sample (very high volume, -1.3; high volume, -0.12 ; controls, -1.3 ).

\section{HORMONE ANALYSES}

There were no significant differences in either total testosterone or free testosterone between groups. The very high volume runners had the lowest mean total and free testosterone when compared with high volume runners and controls (not significant). In all three groups, both total and free testosterone were within the normal range for this population (table 2). The mean free testosterone value for the very high volume runners (54.4 (7.9) pmol/1) fell just above the lower limit for normal.

In the runners, simple correlation showed significant negative associations between weekly training volume and total testosterone $(r=-0.73, \mathrm{p}<0.01)$, and weekly training volume and free testosterone $(r=-0.79$, $\mathrm{p}<0.005$; fig 2). The correlations between free/ total testosterone and BMD at all sites were not significant ( $r$ values ranged from 0.03 to 0.16 , $\mathrm{p}>0.45$ for all relations).

\section{Discussion}

This study is the first to evaluate regional BMD in 40-55 year old distance runners who had trained and competed consistently for a minimum of 20 years. Its design allowed us to observe athletes whose training level was very consistent for nearly half of their lives. High volume distance running, between 64 and 80 $\mathrm{km}$ a week, was positively associated with BMD at the mechanically loaded proximal femur, but not at the lumbar spine. BMD of very high volume runners who ran more than $95 \mathrm{~km}$ a week for over 20 years did not differ from controls. Although lower testosterone levels were significantly related to higher training volumes, the hormone levels did not explain the between group differences in BMD.

\section{PROXIMAL FEMUR}

Training volume (high volume runners $v$ controls) Our observation of higher proximal femur $\mathrm{BMD}$ in the high volume runners is consistent with two other cross sectional studies that examined BMD of the lower extremity in younger runners performing similar training volumes. About 10\% higher proximal femur BMD was observed in adult male runners (mean age 32 years) averaging about $70 \mathrm{~km} \mathrm{a}$ week than in age matched controls. ${ }^{10}$ Femur

Table 2 Mean total testosterone and free testosterone for very high volume runners, high volume runners, and controls

\begin{tabular}{|c|c|c|c|c|}
\hline & Normal range & Very high volume & High volume & Controls \\
\hline $\mathrm{n}$ & & 5 & 7 & 12 \\
\hline Total testosterone $(\mathrm{nmol} / \mathrm{l})$ & $10.4-38.2$ & $15.1(2.6)[12.9-19.6]$ & $22.8(3.6)[21.0-30.8]$ & $18.2(7.1)[12.2-37.0]$ \\
\hline Free testosterone $(\mathrm{pmol} / \mathrm{l})$ & $50.0-130$ & $54.4(7.9)[48.0-68.0]$ & $69.0(8.3)[58.0-80.0]$ & $73.7(27.3)$ [36.0-117] \\
\hline
\end{tabular}

Data are expressed as mean (SD) [range]. Normal range for hormone concentrations taken from Arce and De Souza. ${ }^{40}$ No significant differences observed between groups. 


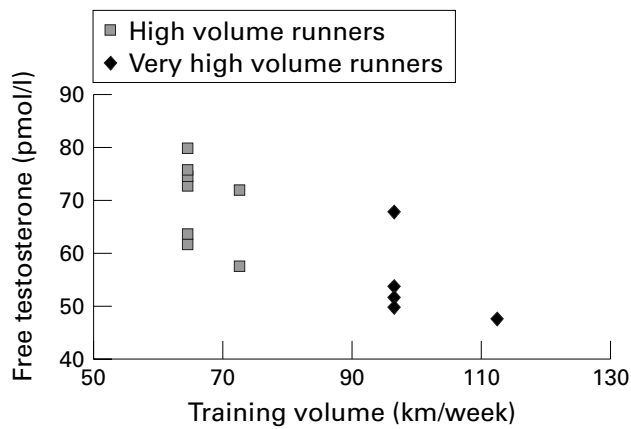

Figure 2 Scatterplot of free testosterone levels (pmol/l) $v$ running volume for 12 distance runners. $r=-0.79$, $p<0.005$.

(proximal femoral neck to knee joint) BMD in male endurance track athletes between the ages of 17 and 26, training an average of 10.9 (5.1) hours a week, was $6.75 \%$ higher than in sedentary controls. ${ }^{8}$ However, MacDougall et a ${ }^{23}$ reported significantly higher lower leg (tibia + fibula) BMD in runners (aged 20-45) not exceeding $32 \mathrm{~km}$ a week compared with two control groups (active and sedentary men). There was a trend towards lower BMD in runners training between 32 and $88 \mathrm{~km}$ a week, and the BMD for this group was higher than that of the sedentary controls, only.

\section{Training volume (very high volume runners $v$} controls)

Our finding that the proximal femur BMD of the very high volume runners ( $>95 \mathrm{~km}$ a week) did not differ significantly from that of the control group is similar to the report by MacDougall et $a l^{23}$ on runners training over $100 \mathrm{~km}$ a week. Hetland et $a l^{11}$ reported lower BMC at the trochanteric region in runners training at least $100 \mathrm{~km}$ a week than in non-running active controls. However, the BMC of these runners was not lower than that of controls at the femoral neck, an area that responds to compressive loading to a greater extent than the trochanter. ${ }^{24}$ In their study, the negative association between training volume and trochanteric BMC remained significant when the model was corrected for body size. ${ }^{11}$

Training volume (threshold)

The results of this study, and others that evaluated proximal femur BMD in distance runners $^{1123}$ training at high (up to about $90 \mathrm{~km}$ a week) compared with very high $(>90 \mathrm{~km}$ a week) volumes suggest that a volume threshold exists. Animal experiments also suggest that above a certain load frequency no further increases are observed in BMC and BMD. ${ }^{5}{ }^{6} \mathrm{In}$ the classic studies by Lanyon, ${ }^{5} 36$ loading cycles a day were as effective as 360 or 1800 in increasing $\mathrm{BMC}$ in turkey ulnae. Umemura and colleagues ${ }^{6}$ showed that five jumps a day were as effective as $40-100$ jumps in increasing BMC. The present study suggests that a positive training response is obtained when the training volume is below $95 \mathrm{~km}$ a week.

Influence of age/training years

$\operatorname{Age}^{25}$ and, possibly, years of continued training $^{26}$ may be as important as training volume for proximal femur BMD in older athletes, underlining the importance of considering all three of these variables. The strengths of this study are that, firstly, subjects had maintained consistent training volumes for a minimum of 20 years, and secondly, subjects were all aged between 40 and 55 years. Limited comparisons can be made between our study and others measuring more widespread age groups of male runners, whose training volume and training years vary considerably. ${ }^{927}$

In both their five ${ }^{28}$ and nine ${ }^{9}$ year longitudinal studies, Lane et al suggested that training volume must be maintained to keep the higher BMD noted in aging runners compared with controls. Although both runners and controls lost bone mineral over time, the loss was greatest in those runners who decreased their running volumes substantially. ${ }^{28}$ Runners in our study maintained high training volumes for more than 20 years, and this may have contributed to the observed differences in BMD.

The net gain of bone that results from modelling in response to high mechanical loads or strain occurs primarily during growth, ${ }^{29}{ }^{30}$ and "starting age" of training can substantially influence peak BMC. ${ }^{31}$ Five of the seven high volume runners had commenced vigorous training before the age of 18 , while only one of the very high volume runners had done so before 18 . Thus the high volume runners may have had a higher BMD when they reached adulthood. The design of this study did not permit us to evaluate the specific influence of childhood activity on adult bone status.

LUMBAR SPINE

We observed no difference in lumbar spine BMD among groups, and this is consistent with the report from Goodpaster and colleagues. ${ }^{27}$ Their group found no difference in BMD between middle aged, previously elite (previously training between 80 and $150 \mathrm{~km}$ a week) distance runners currently training at various levels compared with normative values. However, others have found lower vertebral bone mineral in young adult runners training over about $92 \mathrm{~km}$ a week, as compared with controls. ${ }^{112}$ One study suggested a positive association between low volumes of distance running, about $43 \mathrm{~km}$ a week, and lumbar spine BMD. ${ }^{9}$ Potential mechanisms that may explain our results relate to the biomechanical forces associated with running and the endocrine regulation of bone mass.

\section{Biomechanics of loading: site specificity}

We observed the greatest bone benefit at the primary weight bearing sites-that is, lower limb-which is consistent with the mechanostat theory. ${ }^{32}$ Jogging elicits peak forces at the hip in the range of five to six times body weight, ${ }^{33}$ but these forces are attenuated as they propagate into the lumbar spine. ${ }^{34}$ Bennell et al $l^{8}$ reported diminishing BMD differences between track athletes and controls at skeletal sites at increasing distances from the foot. There were no differences at the lumbar spine. ${ }^{8}$ Furthermore, BMD increases are more likely to occur under unusual strains of uneven 
distribution, than under the repetitive low amplitude strains associated with running or other daily physical activities. ${ }^{35}$

Our controls were recreationally active healthy men, who participated in weight bearing non-endurance exercise at least twice a week, and not purely sedentary men. This may have tended to equalise our groups in terms of loading at the lumbar spine and thus limit BMD differences at this site. Although the controls performed less activity overall than the runners, they participated more often in activities such as weight lifting, tennis, and heavy yard work. It is likely that these exercises represented unique stresses to the skeleton, which could load the lumbar spine as much as the repetitive reaction forces induced in distance running. ${ }^{36}$

TESTOSTERONE

In our study, there was a negative correlation between weekly running volume and both total and free testosterone; however, the within group variances were high. This, and the relatively small sample size, may partially explain the non-significant differences in both free and total testosterone between groups. However, the lowest absolute value for both measures belonged to the very high volume runners. This group had a mean free testosterone level just above the lower limit of the physiological normal range. In several previous studies, distance runners (training more than $95 \mathrm{~km}$ a week) had lower resting testosterone levels than less active men. ${ }^{15-17}$ Future studies with serial blood sampling over time may have greater power to detect differences in testosterone levels between groups of distance runners.

TESTOSTERONE/BONE RELATION

In our study and in others, ${ }^{14-18} 37$ the reported testosterone levels in distance runners fell within the normal healthy range. In two studies, BMD of chronically hypogonadal men (with testosterone levels below the normal range, as in Klinefelter's syndrome and hypogonadotropic hypogonadism) was significantly lower than that of men who had normal testosterone levels. ${ }^{38}{ }^{39}$ Although researchers report differences in testosterone levels when comparing less active controls with distance runners, ${ }^{15-17}$ the testosterone-bone relation remains clinically insignificant when normal levels of testosterone are maintained.

\section{CLINICAL IMPLICATIONS}

(a) Both high and very high volume running are associated with normal BMD.

(b) High (but not very high) running volumes are associated with higher BMD than that of non-athletic controls, thereby conferring a slightly decreased risk of osteoporotic fracture. (c) Although testosterone levels were negatively associated with training volume, most fell within the normal range and were not related to BMD.

CONCLUSIONS

In this study of 40-55 year old men, running $64-80 \mathrm{~km}$ a week was associated with proximal femur BMD that exceeded that of active controls. However, running more than $95 \mathrm{~km}$ a week was associated with BMD equivalent to active controls. Although we observed a negative association between free testosterone and training volume in this study of healthy male adults, this did not explain the differences in BMD between groups.

This project was funded by The Vancouver Medical Services Foundation (grant no BCM97-0137). We thank the staff at Fairmont Bone Density as well as the technicians at Clinical Chemistry, Vancouver Hospital for analysing the blood samples. Contributors: K M initiated the study, formulated the initial hypotheses, and coordinated all aspects of data collection, analysis, and writing of the paper. J T refined the hypotheses, ansisted in subject recruitment, collected blood samples for horassisted in subject recruitment, collected blood samples for hor-
mone analysis, and edited initial manuscripts. H Mc directed mone analysis, and edited initial manuscripts. H Mc directed the analysis and discussion of BMD measurements, and worked
closely with $\mathrm{K} \mathrm{M}$ in the writing of the paper. H Mc is the guarclosely with $\mathrm{K} \mathrm{M}$ in the writing of the paper. $\mathrm{H} \mathrm{Mc}$ is the guar-
antor for this study. K K stimulated discussion of the results and antor for this study. K K stimulat
edited the paper extensively.

1 Suominen H, Rahkila P, Era P, et al. Functional capacity in middle-aged endurance and power athletes. In: Harris R, Harris S, eds. Physical activity, aging and sports. Vol I. Scientific and medical research.Albany, NY: Center for the Study of Aging, 1989:213-18.

2 Conroy B, Kraemer W, Maresh C, et al. Bone mineral density of elite junior Olympic weightlifters. Med Sci Sports Exerc 1993;25:1103-9.

3 Haapasalo H, Sievanen H, Kannus P, et al. Dimensions and estimated mechanical characteristics of the humerus after long term tennis loading. F Bone Miner Res 1996;11;86472.

4 Mosley J, March B, Lynch J, et al. Strain magnitude changes in whole bone architecture in growing rats. Bone 1997;20: $191-8$.

5 Lanyon L. Functional strain as a determinant for bone remodeling. Calcif Tissue Int 1984;36(suppl 1):S56-61.

6 Umemura Y, Ishiko T, Yamauchi T, et al. Five jumps per day Umemura Y, Ishiko T, Yamauchi T, et al. Five jumps per day Res 1997;12:1480-5.

7 Hamdy R, Anderson J, Whalen K, et al. Regional differences in bone density of young men involved in different exercises. Med Sci Sports Exerc 1994;26:884-8.

8 Bennell K, Malcolm S, Khan K, et al. Bone mass and bone turnover in power athletes, endurance athletes, and controls: a 12 month longitudinal study. Bone 1997;20: $477-84$

9 Lane N, Oehlert J, Bloch D, et al. The relationship of running to osteoarthritis of the knee and hip and bone mineral density of the lumbar spine: a 9 year longitudinal study. F Rheumatol 1998;25:334-41.

10 Brahm H, Strom H, Piehl-Aulin K. Bone metabolism in endurance trained athletes: a comparison to populationbased controls based on DXA, SXA, quantitative ultrasound and biochemical markers. Calcif Tissue Int 1997;61: 448-54.

11 Hetland M, Haarbo J, Christiansen C, et al. Low bone mass and high bone turnover in male long distance runners. $\mathcal{F}$ Clin Endocrinol Metab 1993;77:770-5.

12 Bilanen J, Blanchard M, Russek-Cohen E. Lower vertebral bone density in male long distance runners. Med Sci Sports Exerc 1989;21:66-70

13 De Souza MJ, Miller BE. The effect of endurance training on reproductive function in male runners: a "volume threshold" hypothesis. Sports Med 1997;23:357-74

14 MacConnie S, Barkan A, Lampman M, et al. Decreased hypothalamic gonadotropin-releasing hormone secretion in male marathon runners. N Engl F Med 1986;315:411-17.

15 Hackney A, Sinning W, Bruot B. Hypothalamic-pituitarytesticular axis function in endurance-trained males. Int $\mathcal{F}$ Sports Med 1990;11:298-303.

16 Hackney A, Sinning W, Bruot B. Reproductive hormone profiles of endurance-trained and untrained males. Med Sci profts Exerc 1988;20:60-5.

17 Arce JC, De Souza MJ, Pescatello L, et al. Subclinical alterations in hormone and semen profile in athletes. Fertil Steril tions in hormone

18 Wheeler G, Wall S, Belcastro A, et al. Reduced serum testosterone and prolactin levels in male distance runners. fAMA 1984;252:514-16.

19 Vandershueren D, Bouillon R. Androgens and bone. Calcif Tissue Int 1995;56:341-6.

20 Technical Insert. Chiron Diagnostics ACS: 180 Testosterone, 1996.

21 Technical Insert. DPC's Coat-a-Count Free Testosterone Assay, March, 1997.

22 Canadian Physical Activity, Fitness, and Lifestyle Appraisal (CPAFLA) manual. Ottawa: Canadian Society for Exercise Physiology, 1998.

23 MacDougall J, Webber C, Martin J, et al. Relationship among running mileage, bone density, and serum testosterone in male runners. $\mathcal{F}$ Appl Physiol 1992;73:1165-70.

24 Carter DR, Van Der Meulen MCH, Beaupre GS. Skeletal development: mechanical consequences of growth, aging development: mechanical consequences of growth, aging
and disease. In Marcus R, Feldman D, Kelsey J, eds. Osteoporosis. San Diego: Academic Press, 1996:333-50. 
25 Bendavid EJ, Shan J, Barrett-Connor E. Factors associated with bone mineral density in middle-aged men. $\mathcal{F}$ Bone Miner Res 1996;11:1185-90.

26 Karlsson MK, Hasserius R, Obrant KJ. Bone mineral density in athletes during and after career: a comparison between loaded and unloaded skeletal regions. Calcif Tissue Int 1996;59:245-8.

27 Goodpaster BH, Costill DL, Trappe SW, et al. The relationship of sustained exercise training and bone mineral density in aging male runners. Scand F Med Sci Sports 1996:6:21621.

28 Michel BA, Lane NE, Bjorkengren A, et al. Impact of running on lumbar bone density: a 5-year longitudinal study. F Rheumatol 1992;19:1759-63.

29 McKay HA, Petit MA, Schutz RW, et al. Augmented trochanteric bone mineral density after modified physical education classes: a randomized, school-based exercise intervention study in pre- and early-pubescent children. $\mathcal{F}$ Pediatr 2000;136:156-62.

30 Bailey DA, McKay HA, Mirwald RL, et al. The University of Saskatchewan Bone Mineral Accrual Study: a six year lonSaskatchewan Bone Mineral Accrual Study: a six year lon-
gitudinal study of the relationship of physical activity to gitudinal study of the relationship of physical activity to
bone mineral accrual in growing children. F Bone Miner Res bone mineral accruct

31 Kannus P, Haapasalo H, Sankelo M, et al. Effect of starting age of physical activity on bone mass in the dominant arm of tennis and squash players. Ann Intern Med 1995;123:27-31.
32 Frost HM. Bone mass and the mechanostat: a proposal. Anat Rec 1987;219:1-9.

33 Bergmann G, Graichen F, Rohlmann A. Hip joint loading during walking and running, measured in two patients. $\mathcal{f}$ Biomech 1993;26:969-90.

34 Wosk J, Voloshin A. Wave attenuation in skeletons of young healthy persons. F Biomech 1981;14:261-7.

35 Lanyon L. Using functional loading to influence bone mass and architecture: objectives, mechanisms, and relationship with estrogen of the mechanical adaptive process in bone. Bone 1996;18(suppl 1):S37-43.

36 Kohrt W, Ehsani A, Birge S. Effects of exercise involving predominantly either joint-reaction or ground-reaction forces on bone mineral density in older women. 7 Bone Miner Res 1997;12:1253-61.

37 Smith R, Rutherford O. Spine and total body bone mineral density and serum testosterone levels in male athletes. Eur f Appl Physiol 1993;67:330-4.

38 Horowitz $\mathrm{M}$, Wishart J, O'Loughlin $\mathrm{P}$, et al. Osteoporosis and Klinefelter's syndrome. Clin Endocrinol 1992;36:113-18.

39 Finkelstein J, Klibanski A, Neer R, et al. Osteoporosis in men with idiopathic hypogonadotropic hypogonadism. Ann Intern Med 1987;106:354-61.

40 Arce J, De Souza MJ. Exercise and male factor infertility. Sports Med 1993;15:146-69.

\section{Take home message}

Moderate long term distance running in the range of $64-80 \mathrm{~km}$ a week has a positive influence on $\mathrm{BMD}$ of the proximal femur, but higher volumes of running do not elicit the same benefit in middle aged men. Although testosterone levels are negatively related to training volume, they do not predict BMD in distance runners and healthy active controls.

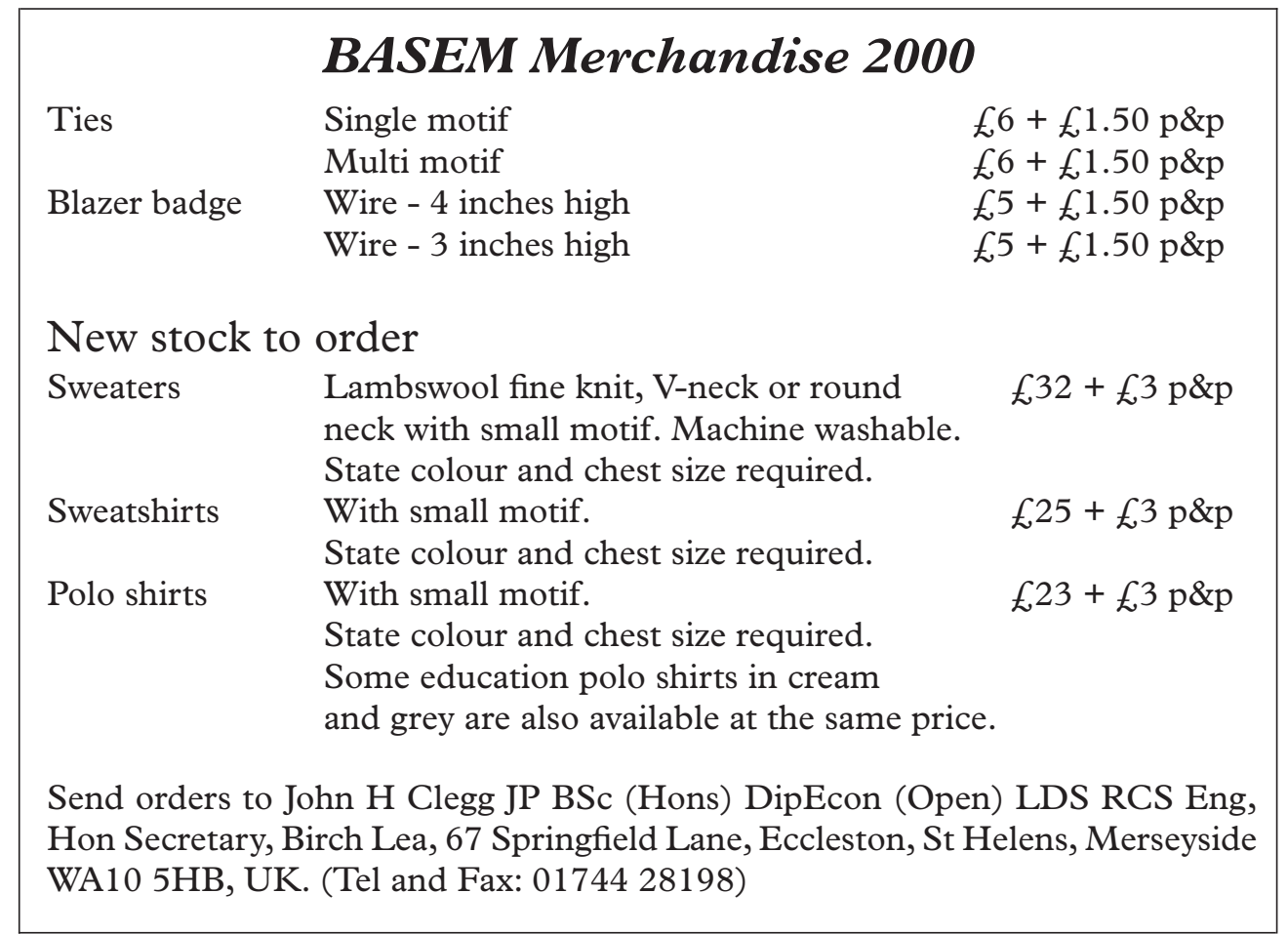

